\title{
Dasatinib induces lung vascular toxicity and predisposes to pulmonary hypertension
}

\author{
Christophe Guignabert, ${ }^{1,2,3}$ Carole Phan, ${ }^{1,2,3}$ Andrei Seferian, ${ }^{1,2,3}$ Alice Huertas, ${ }^{1,2,3}$ Ly Tu, ${ }^{1,2,3}$ Raphaël Thuillet, $^{1,2,3}$ Caroline Sattler, ${ }^{1,2,3}$ \\ Morane Le Hiress, ${ }^{1,2,3}$ Yuichi Tamura, ${ }^{1,2,3}$ Etienne-Marie Jutant,, ${ }^{1,2,3}$ Marie-Camille Chaumais, ${ }^{1,2,3}$ Stéphane Bouchet, ${ }^{4,5}$ \\ Benjamin Manéglier, ${ }^{6}$ Mathieu Molimard, ${ }^{4,5}$ Philippe Rousselot, ${ }^{7,8}$ Olivier Sitbon, ${ }^{1,2,3}$ Gérald Simonneau, ${ }^{1,2,3}$ \\ David Montani, ${ }^{1,2,3}$ and Marc Humbert ${ }^{1,2,3}$ \\ IINSERM UMR S 999, Hôpital Marie Lannelongue, Le Plessis-Robinson, France. ${ }^{2}$ Université Paris-Sud and Université Paris-Saclay, Kremlin-Bicêtre, France. ${ }^{3}$ Assistance Publique - Hôpitaux de Paris, \\ Service de Pneumologie, Centre de Référence de l'Hypertension Pulmonaire Sévère, Département Hospitalo-Universitaire Thorax Innovation, Hôpital de Bicêtre, Kremlin-Bicêtre, France. \\ ${ }^{4}$ Département de Pharmacologie, Centre Hospitalier Universitaire de Bordeaux, Bordeaux, France. ${ }^{5}$ Université de Bordeaux, INSERM U1219, Bordeaux, France. ${ }^{6}$ Service de Biologie Médicale \\ and 'Service d'Hématologie et d'Oncologie, Centre Hospitalier de Versailles, Le Chesnay, France. ${ }^{8}$ Université de Versailles-Saint-Quentin-en-Yvelines, Versailles France.
}

Pulmonary arterial hypertension (PAH) is a life-threatening disease that can be induced by dasatinib, a dual Src and BCR-ABL tyrosine kinase inhibitor that is used to treat chronic myelogenous leukemia (CML). Today, key questions remain regarding the mechanisms involved in the long-term development of dasatinib-induced PAH. Here, we demonstrated that chronic dasatinib therapy causes pulmonary endothelial damage in humans and rodents. We found that dasatinib treatment attenuated hypoxic pulmonary vasoconstriction responses and increased susceptibility to experimental pulmonary hypertension (PH) in rats, but these effects were absent in rats treated with imatinib, another BCR-ABL tyrosine kinase inhibitor. Furthermore, dasatinib treatment induced pulmonary endothelial cell apoptosis in a dose-dependent manner, while imatinib did not. Dasatinib treatment mediated endothelial cell dysfunction via increased production of ROS that was independent of Src family kinases. Consistent with these findings, we observed elevations in markers of endothelial dysfunction and vascular damage in the serum of CML patients who were treated with dasatinib, compared with CML patients treated with imatinib. Taken together, our findings indicate that dasatinib causes pulmonary vascular damage, induction of ER stress, and mitochondrial ROS production, which leads to increased susceptibility to PH development.

\section{Introduction}

Pulmonary arterial hypertension (PAH) is a severe progressive cardiopulmonary disorder that carries a high mortality rate, for which there is no cure at this time (1). Pulmonary endothelial cell (EC) injury and dysfunction as well as aberrant vascular wall cell accumulation are strongly suspected to be key contributing factors in initiating and mediating pulmonary vascular remodeling in human PAH (2-7). In addition, it is now well established that a complex interplay of genetic and environmental factors facilitates the onset and progression of the disease (8).

$\mathrm{PAH}$ can be induced by exposure to drugs or toxins, particularly to appetite suppressant drugs (i.e., aminorex, fenfluramine derivatives, and benfluorex), amphetamines, and dasatinib, an oral ATP-competitive inhibitor of tyrosine kinases (TKI) $(8,9)$. Dasatinib is presently approved for first-line treatment of Philadelphia-positive

Conflict of interest: In the past 3 years, D. Montani has served as a consultant for Actelion, Bristol-Myers Squibb (BMS), Bayer, GlaxoSmithKline (CSK), Novartis, and Pfizer. In the past 3 years, $\mathrm{O}$. Sitbon has served as a consultant for Actelion, Bayer, CSK, and Pfizer. In the past 3 years, G. Simonneau has served as a consultant for Actelion, Bayer, CSK, and Pfizer. In the past 3 years, M. Humbert has served as a consultant for Actelion, Bayer, CSK, Novartis, and Pfizer. P. Rousselot has received a research grant from BMS. M. Molimard has served as a consultant for Allergologisk Laboratorium København, BMS, CSK, Novartis, Pfizer, and Stallergen.

Submitted: December 28, 2015; Accepted: May 26, 2016.

Reference information: / Clin Invest. 2016;126(9):3207-3218. doi:10.1172/JCI86249. chronic myelogenous leukemia (CML) and for all phases of Philadelphia-positive CML with resistance or intolerance to prior therapy, including imatinib. Dasatinib is a second-generation TKI that is more potent than imatinib on BCR-ABL, c-Kit, and PDGFR, and, in contrast to imatinib, on Src family kinases (10). The lowest incidence of PAH occurring in patients exposed to dasatinib in France was estimated in 2012 at $0.45 \%$ (9). Notably, rapid clinical, functional, or hemodynamic improvements are often observed within 4 months of dasatinib discontinuation (9). These observations support the notion that dasatinib-induced $\mathrm{PAH}$ may be at least in part reversible. In addition, the specific cellular events and signaling pathways that trigger the structural and functional pulmonary vascular remodeling are unknown. As a result, we lack effective marker-based screening strategies and mechanism-based treatment options for PAH occurring in dasatinib-treated patients. In addition, a better understanding of mechanisms involved in dasatinib-induced PAH would not only help to ameliorate the management of dasatinib-treated patients, but also would improve our knowledge about the pathomechanisms underlying PAH development.

In this study, we determined whether (a) chronic treatment of rats with dasatinib causes changes in pulmonary hemodynamics and pulmonary vascular structures; (b) pretreatment of rats with dasatinib exaggerates the response to monocrotaline (MCT) administration and exposure to chronic hypoxia $(\mathrm{CHx}), 2$ inducers of pulmonary vascular remodeling; (c) dasatinib treatment causes 
A

Group A:

Chronic treatment with dasatinib, imatinib, or vehicle for 4 wks
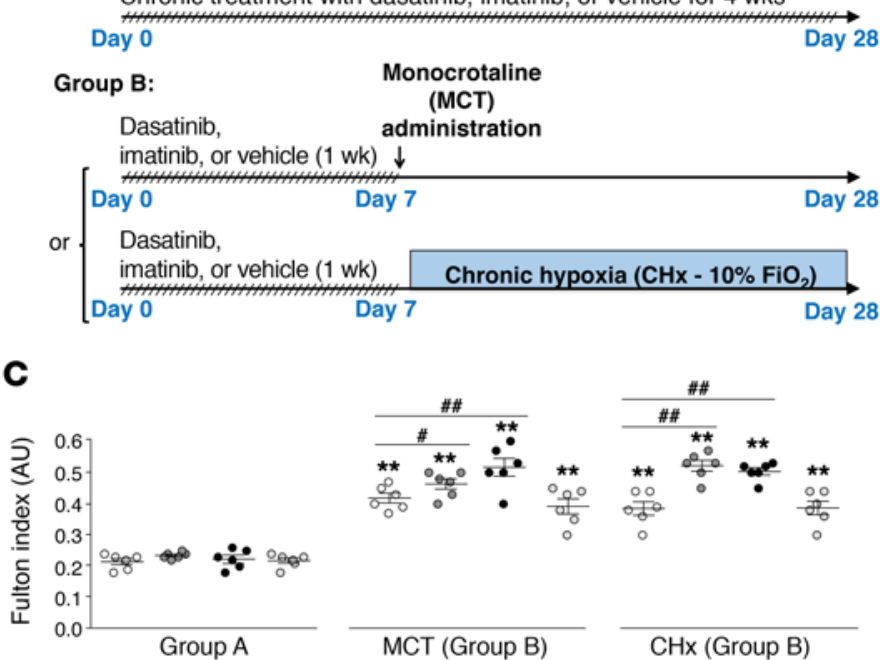

$\mathrm{CHx}$ (Group B)

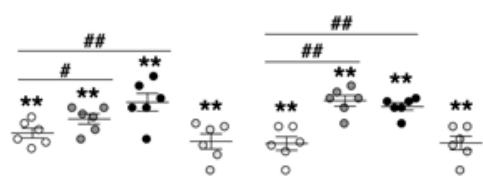

ner Gement

E

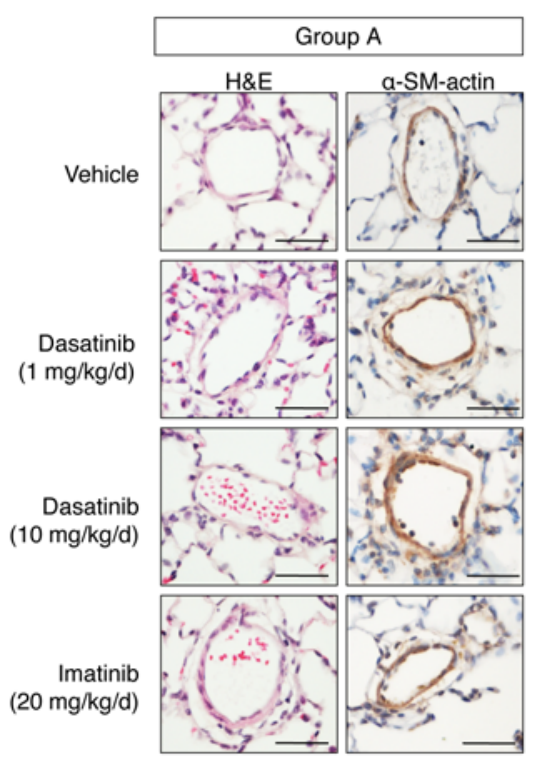

- Dasatinib, $10 \mathrm{mg} / \mathrm{kg} / \mathrm{d}$

O Dasatinib, $1 \mathrm{mg} / \mathrm{kg} / \mathrm{d} \quad$ Imatinib, $20 \mathrm{mg} / \mathrm{kg} / \mathrm{d}$

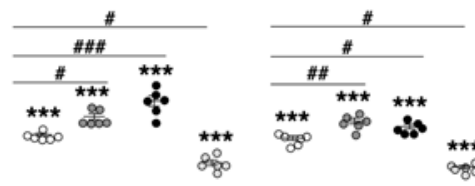

MCT (Group B)

$\mathrm{CHx}$ (Group B)
D

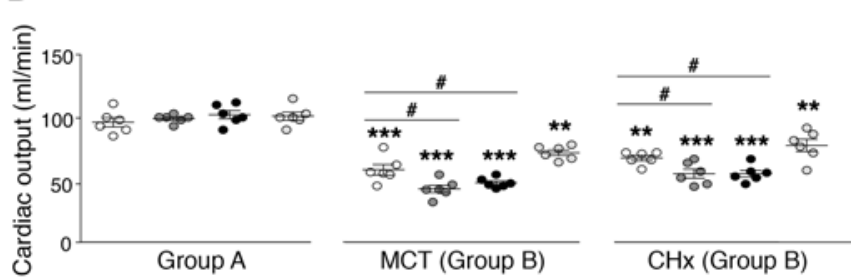

$\mathbf{F}$

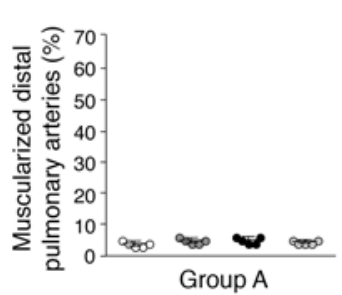

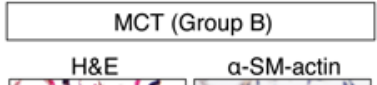
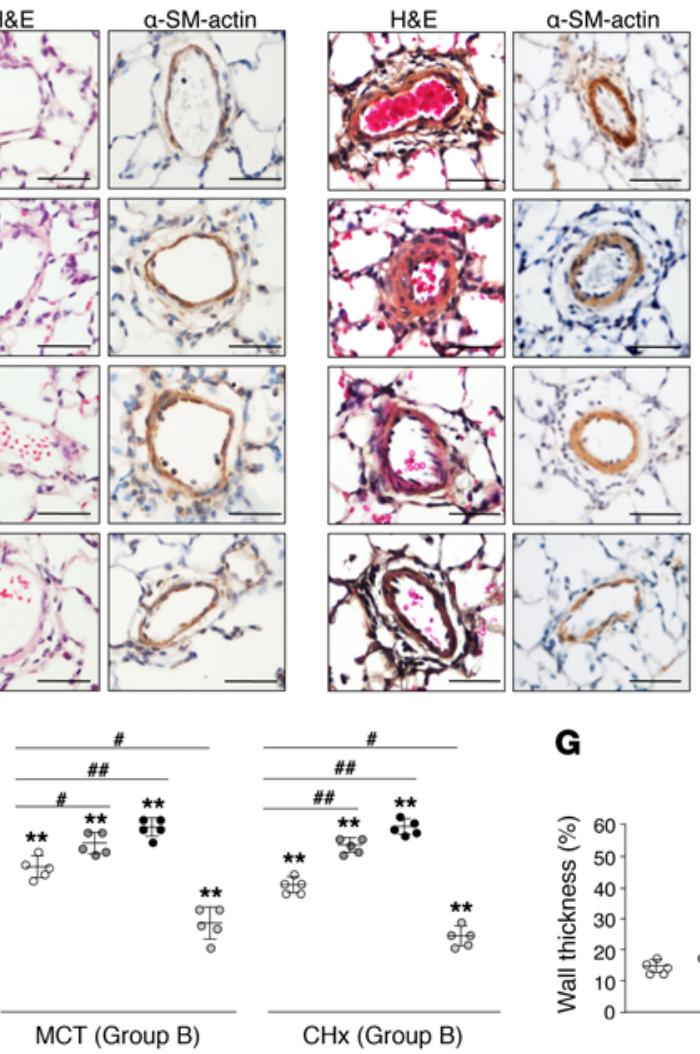

G

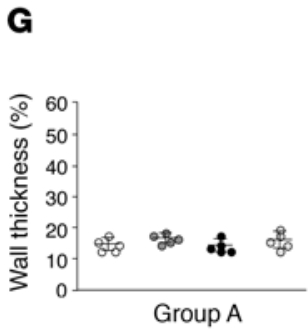

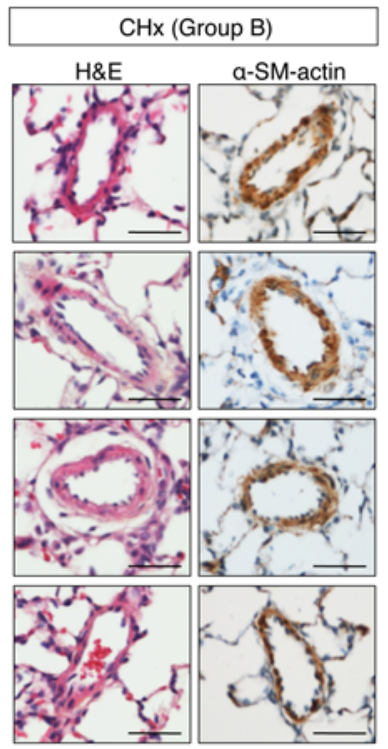

Figure 1. Pretreatment of rats with dasatinib confers an exaggerated response to monocrotaline (MCT) and chronic hypoxia (CHx), $\mathbf{2}$ inducers of experimental pulmonary hypertension in vivo. (A) Rodent models used to study the in vivo effects of dasatinib on pulmonary hemodynamic parameters and vascular remodeling: chronic treatment group (group A) and pretreatment group (group B). (B-D) Values of mean pulmonary arterial pressure (PAP) (B), Fulton index (C), and cardiac output (D) in vehicle-, dasatinib-, and imatinib-treated rats. (E) Representative images of H\&E- and $\alpha$-smooth muscle (SM) actin-stained sections of distal pulmonary arteries in vehicle-, dasatinib-, and imatinib-treated rats. (F and G) Quantification of the percentage of muscularized pulmonary arteries (F) and wall thickness (G) in lungs of vehicle-, dasatinib-, and imatinib-treated rats. Horizontal lines

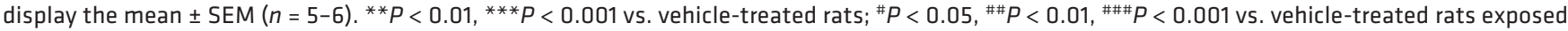
to MCT or to CHx. Scale bars: $20 \mu \mathrm{m}$. 
pulmonary EC dysfunction in vivo and in vitro using primary cultures of human pulmonary ECs; (d) dasatinib-induced pulmonary EC damage/dysfunction involves Src-dependent or -independent mechanisms; (e) dasatinib can lead to the generation of ROS both in vitro and in vivo; and (f) dasatinib treatment may cause pulmonary endothelial dysfunction or vascular damage in humans, via assessment of serum levels of soluble damage markers. In order to answer these questions, imatinib, another TKI used as first-line treatment for leukemia but not associated with PAH, was used as a TKI control in our in vivo and in vitro studies.

\section{Results}

Pretreatment of rats with dasatinib exaggerates the response to MCT administration and exposure to chronic hypoxia, 2 inducers of pulmonary vascular remodeling. To determine the in vivo effects of dasatinib on pulmonary hemodynamic parameters and vascular remodeling, 4-week-old male Wistar rats weighing $100 \mathrm{~g}$ were divided into 2 main groups: group A, the chronic treatment group, in which rats were treated with daily i.p. injections of vehicle (DMSO), dasatinib, or imatinib (as previously described in refs. 11, 12) for 4 weeks (Figure 1A); and group B, the pretreatment group, in which rats were pretreated for 1 week with daily i.p. injections of dasatinib, imatinib, or vehicle and then randomly divided in 2 groups for pulmonary hypertension $(\mathrm{PH})$ induction: MCT injection or chronic hypoxia exposure for 3 weeks (Figure 1A). Prior to this in vivo study, a pharmacokinetic study of dasatinib was performed in rats, indicating that $1 \mathrm{mg} / \mathrm{kg} / \mathrm{d}$ of dasatinib i.p. is close to the clinically relevant dose (Supplemental Figure 1; supplemental material available online with this article; doi:10.1172/JCI86249DS1). For this dose, the peak concentration of dasatinib was observed approximately 1 hour after i.p. administration with a maximum concentration observed $\left(\mathrm{C}_{\max }\right)$ of $92 \pm 21 \mathrm{ng} / \mathrm{ml}$. Furthermore, the value of dasatinib returned to levels similar to the pre-dose after 24 hours (Supplemental Figure 1). On the basis of the above observations and because side effects are very often transient and dose-related, we chose in our study a $1 \times$ and $10 \times$ clinically relevant dose of dasatinib, namely 1 and $10 \mathrm{mg} / \mathrm{kg} / \mathrm{d}$.

We demonstrated that dasatinib-pretreated rats (group B) developed an exaggerated response to MCT- and chronic hypoxia-induced $\mathrm{PH}$, in contrast to the chronic treatment group (group A), in which no hemodynamic changes were observed (Figure 1, $B-G)$. Indeed, we found in dasatinib-pretreated rats, when compared with vehicle- or imatinib-pretreated rats, a substantial increase in hemodynamics (mean pulmonary arterial pressure, or mPAP; Figure 1B), right ventricular hypertrophy (expressed as the Fulton index; Figure 1C), lower cardiac output (Figure 1D), and increased pulmonary vascular remodeling (assessed by the percentage of muscularized vessels and wall thickness; Figure 1, E-G). In addition, we noted an accumulation of $\mathrm{CD}^{+}$(T lymphocytes), $\mathrm{CD}^{2} 5^{+}$(leukocytes), and $\mathrm{CD}^{+} 8^{+}$cells (monocytes/macrophages) in the perivascular area of muscularized vessels in rats chronically treated with dasatinib (group A) compared with vehicle- or imatinib-treated rats (Supplemental Figure 2, left panel). However, we found a significant increase in $\mathrm{CD}^{+}, \mathrm{CD}^{+} 5^{+}$, and $\mathrm{CD}^{+} 8^{+}$cells in rats pretreated with dasatinib and exposed to MCT or chronic hypoxia compared with vehicle- or imatinib-pretreated rats (group B; Supplemental Figure 2, middle and right panels).
Taken together, our results showed that dasatinib at a clinically relevant dose $(1 \times)$ does not lead to $\mathrm{PH}$, but can predispose to its development, a phenomenon more pronounced with the high dose of dasatinib (10x) and not observed with imatinib. These observations strongly support the notion that dasatinib treatment modulates $\mathrm{PH}$-predisposing factors that contribute to the exaggerated MCT- or chronic hypoxia-induced PH in vivo.

Dasatinib treatment causes pulmonary EC dysfunction in vivo. Since pulmonary EC damage/dysfunction is a key pathophysiological mechanism that could be detrimental for disease susceptibility and development of pulmonary vascular remodeling in PAH $(3,5)$, we investigated the in vivo effects of dasatinib on pulmonary endothelial functions.

To this aim, we first examined the effects of vehicle, dasatinib, and imatinib treatment on acute hypoxic pulmonary vasoconstriction (HPV) in vivo, an essential physiological mechanism adapting lung perfusion to regional ventilation that depends critically on the function of the pulmonary endothelium (13). We found that rats treated with dasatinib (10x) exhibited a blunted HPV when compared with rats treated with vehicle, the low dose of dasatinib, or imatinib (Figure 2A). Indeed, we did not find a significant increase in MPAP after acute hypoxia exposure in rats treated with the high dose of dasatinib compared with the other groups, which displayed a quasinormal HPV (Figure 2A).

Furthermore, we found that in vivo chronic dasatinib treatment led to a substantial increase in levels of key serum markers of endothelial dysfunction and damage or of vascular injury named soluble ICAM-1 (sICAM-1), soluble VCAM-1 (sVCAM-1), and soluble E-selectin (sE-selectin) in rats treated with $1 \times$ of dasatinib when compared with imatinib- or vehicle-treated rats, a phenomenon that was even more pronounced at a dose of $10 \times$ (Figure $2 \mathrm{~B}$ ).

One aspect of vascular dysfunction and damage is the EC activation characterized by the expression of adhesion molecules. Confocal microscopic analyses were then used to investigate their protein levels in pulmonary ECs in rat lung specimens. Double labeling with the endothelial marker Tie2 and either ICAM-1, VCAM-1, or E-selectin showed a substantial increased expression of the 3 adhesion molecules in dasatinib-treated rats compared with vehicle- or imatinib-treated rats (Figure 2C).

Since damage of ECs can result from conditions that stress the ER and induce a signaling network termed unfolded protein response (UPR) that can play a potential role in the initiation and progression of the disease (14-18), we next examined whether dasatinib could lead to UPR activation in these rat lung specimens treated with dasatinib when compared with those exposed to imatinib. We found that dasatinib treatment led to an increase in the molecular chaperone glucose-regulated protein 78 (GRP78) in rat lung homogenates (Figure 2D). In addition, our results showed that in vivo treatment with dasatinib led to a significant increase in the expression of the cleaved form of activating transcription factor 6 (ATF6) and X-box-binding protein 1 (XBP1) and to a significant decrease in the phosphorylated form of eukaryotic initiation factor (eIF2 $\alpha$ ) (Figure 2E) compared with vehicle and imatinib treatments. These results strongly suggest that dasatinib treatment induces an abnormal activation of the 3 main UPR-dependent pathways, namely ATF6, protein kinase RNA-like ER kinase (PERK), and inositol-requiring enzyme $1 \alpha$ (IRE1 $\alpha$ ) axes, respectively, and in the chaperone GRP78 involved in these pathways. 
A
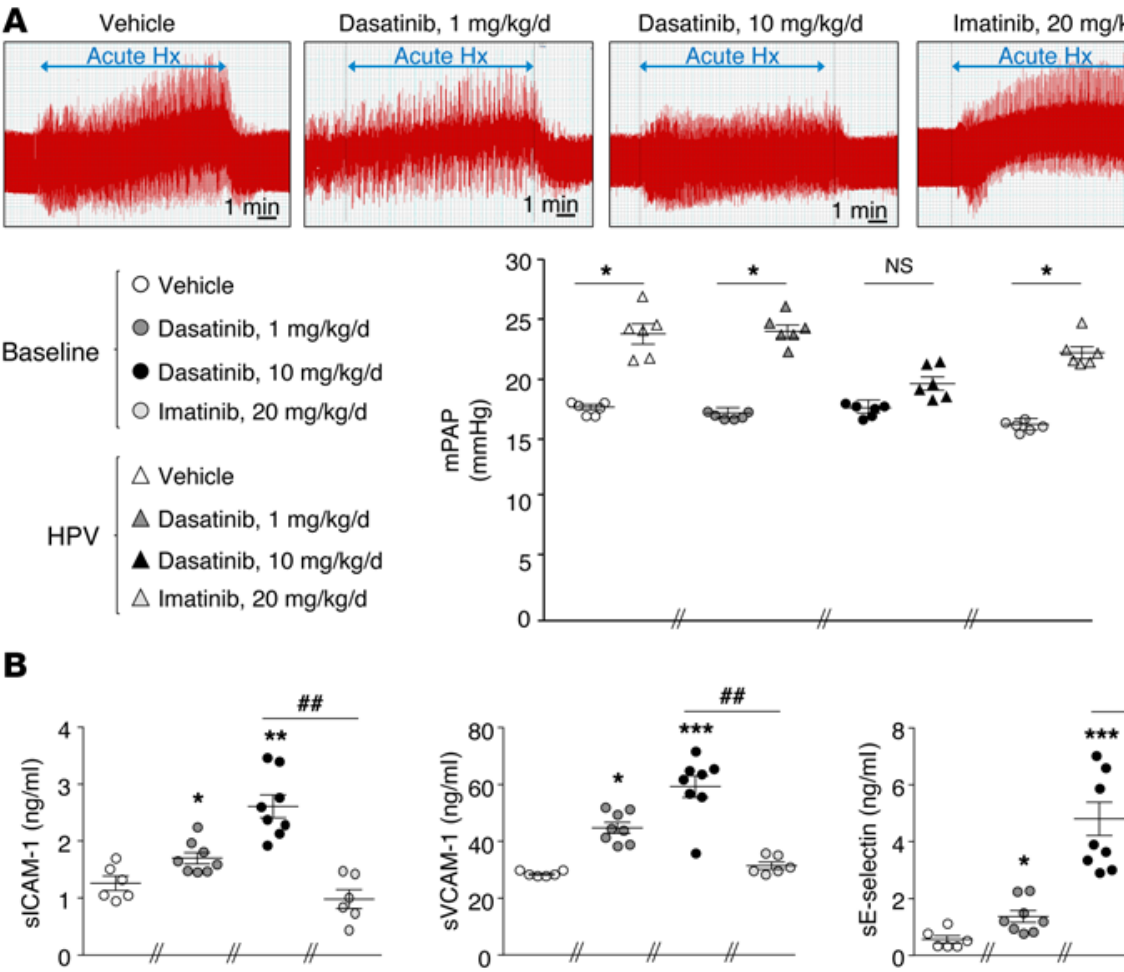

- Dasatinib, $1 \mathrm{mg} / \mathrm{kg} / \mathrm{d}$ o Vehicle

- Dasatinib, $10 \mathrm{mg} / \mathrm{kg} / \mathrm{d}$

Imatinib, $20 \mathrm{mg} / \mathrm{kg} / \mathrm{d}$

HPV $\left\{\begin{array}{l}\Delta \text { Vehicle } \\ \Delta \text { Dasatinib, } 1 \mathrm{mg} / \mathrm{kg} / \mathrm{d} \\ \Delta \text { Dasatinib, } 10 \mathrm{mg} / \mathrm{kg} / \mathrm{d} \\ \Delta \text { Imatinib, } 20 \mathrm{mg} / \mathrm{kg} / \mathrm{d}\end{array}\right.$

B

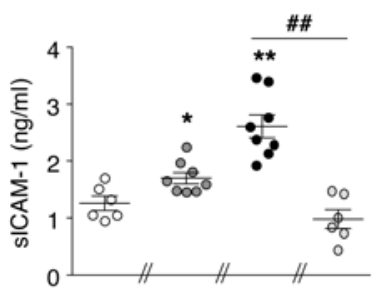

C
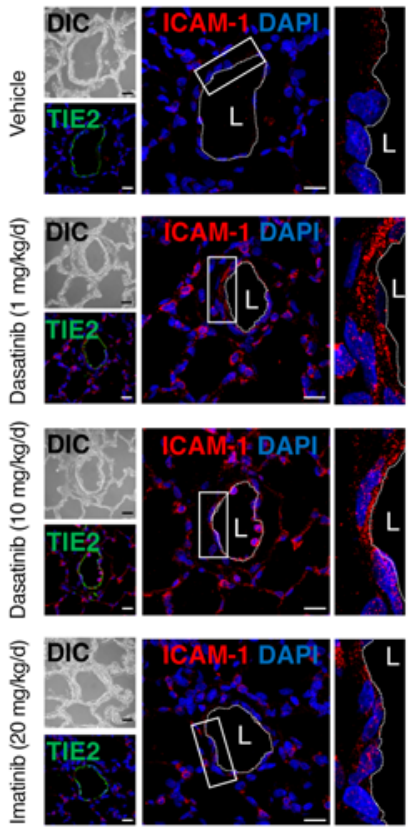
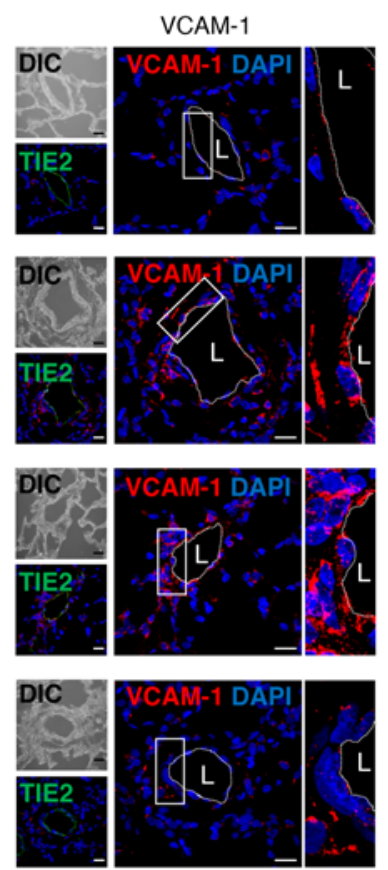
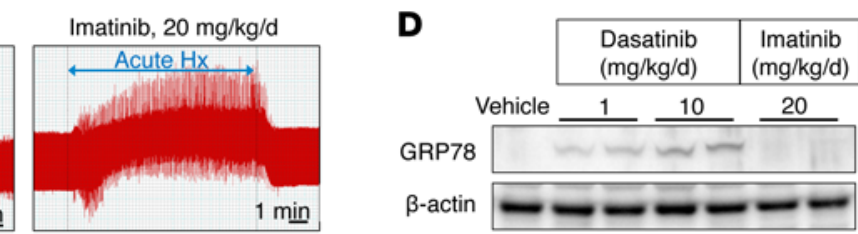

GRP78: $\beta$-actin ratio

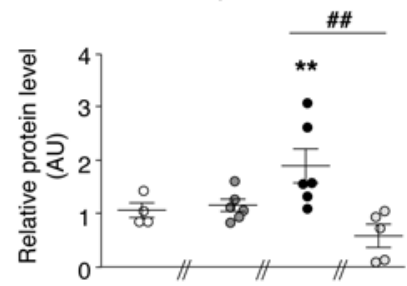

E

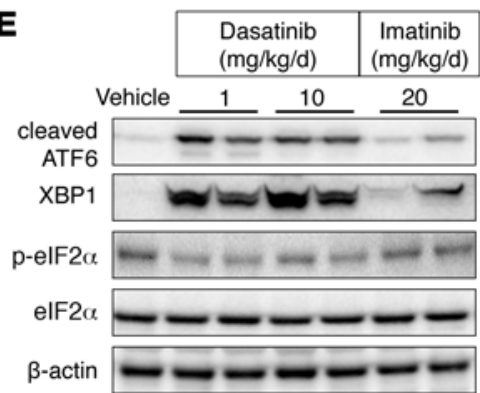

Cleaved ATF6: $\beta$-actin ratio
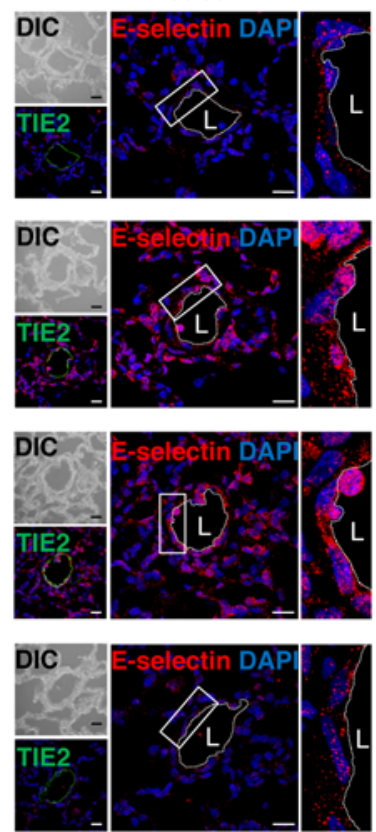

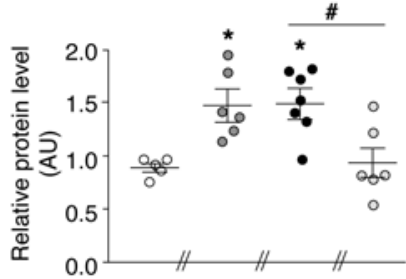

XBP-1: $\beta$-actin ratio

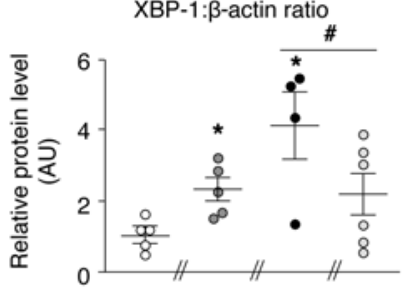

p-elF2 $\alpha$ :elF2 $\alpha$ ratio

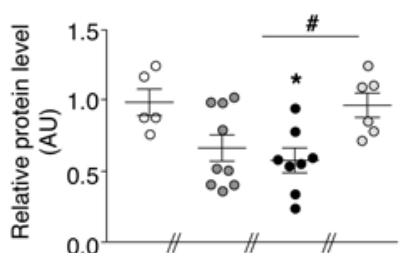

Figure 2. Dasatinib treatment causes pulmonary EC dysfunction in vivo. (A) Values of mean pulmonary arterial pressure (mPAP) in vehicle-, dasatinib-, and imatinib-treated rats while ventilated with room air (baseline) and after 10 minutes of ventilation with a hypoxic gas mixture (acute Hypoxia [Hx]). mPAP increased in rats treated with vehicle, dasatinib (1x), and imatinib, but not in rats treated with dasatinib (10x). (B) Quantification of the circulating levels of soluble forms of ICAM-1 (sICAM-1), VCAM-1 (sVCAM-1), and E-selectin (sE-selectin) in vehicle-, dasatinib-, and imatinib-treated rats. (C) Confocal microscopic analyses and double labeling with ICAM-1, VCAM-1, and E-selectin with the endothelial-specific marker Tie2 in lungs of vehicle-, dasatinib-, and imatinib-treated rats. ( $\mathbf{D}$ and $\mathbf{E}$ ) Representative Western blots and quantification of the glucose-regulated protein 78 (GRP78)/ $\beta$-actin ratio (D) and of the cleaved activating transcription factor 6 (ATF6)/ $\beta$-actin ratio, the X-box-binding protein 1 (XBP1)/ $\beta$-actin ratio, and the phospho-eukaryotic initiation factor $2 \alpha$ (elF2 $\alpha$ )/elF2 $\alpha$ ratio (E) in lungs of vehicle-, dasatinib-, and imatinib-treated rats. Horizontal lines display the mean \pm SEM $(n=4-9)$. ${ }^{*} P<0.05,{ }^{* *} P<0.01,{ }^{* *} P<0.001$ vs. vehicle-treated rats or baseline; ${ }^{\#} P<0.05,{ }^{\#} P<0.01$ vs. rats treated with dasatinib (10x). Scale bars: $20 \mu \mathrm{m}$. HPV, hypoxic pulmonary vasoconstriction; L, lumen. 
A

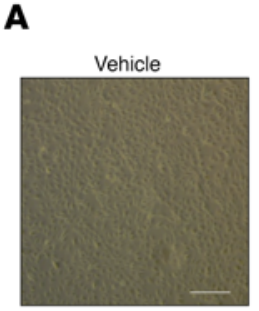

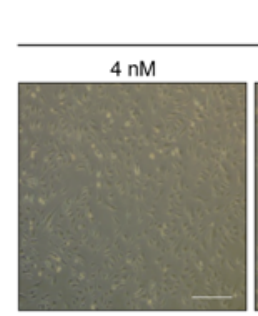

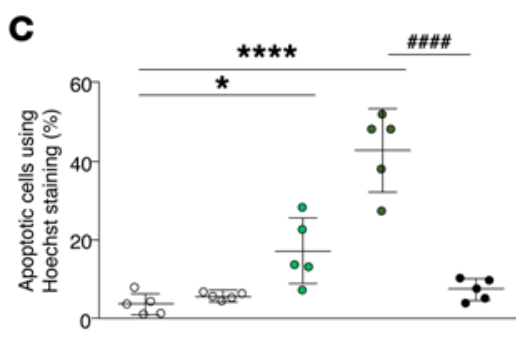

D

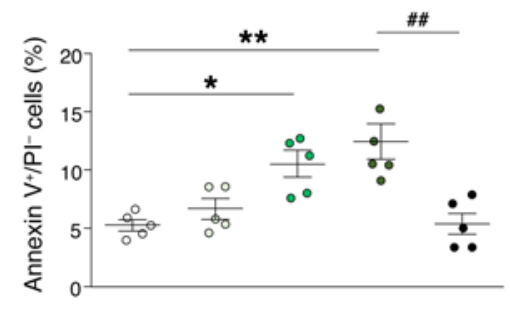

E

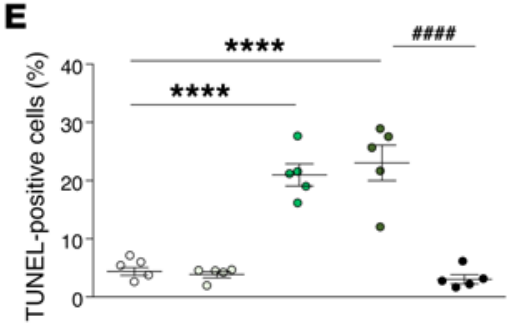

Dasatinib

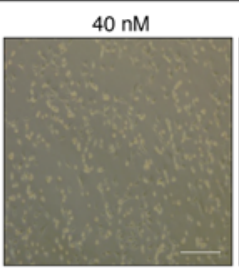

$400 \mathrm{nM}$

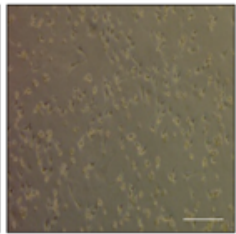

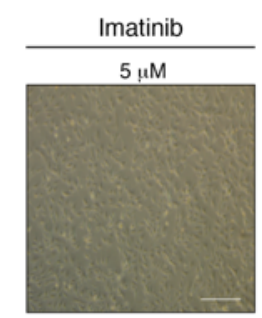

B

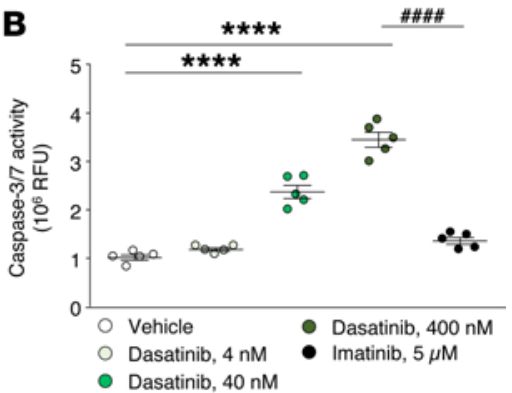

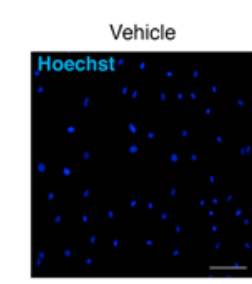

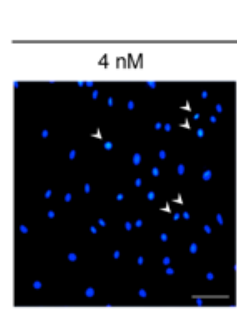

Dasatinib
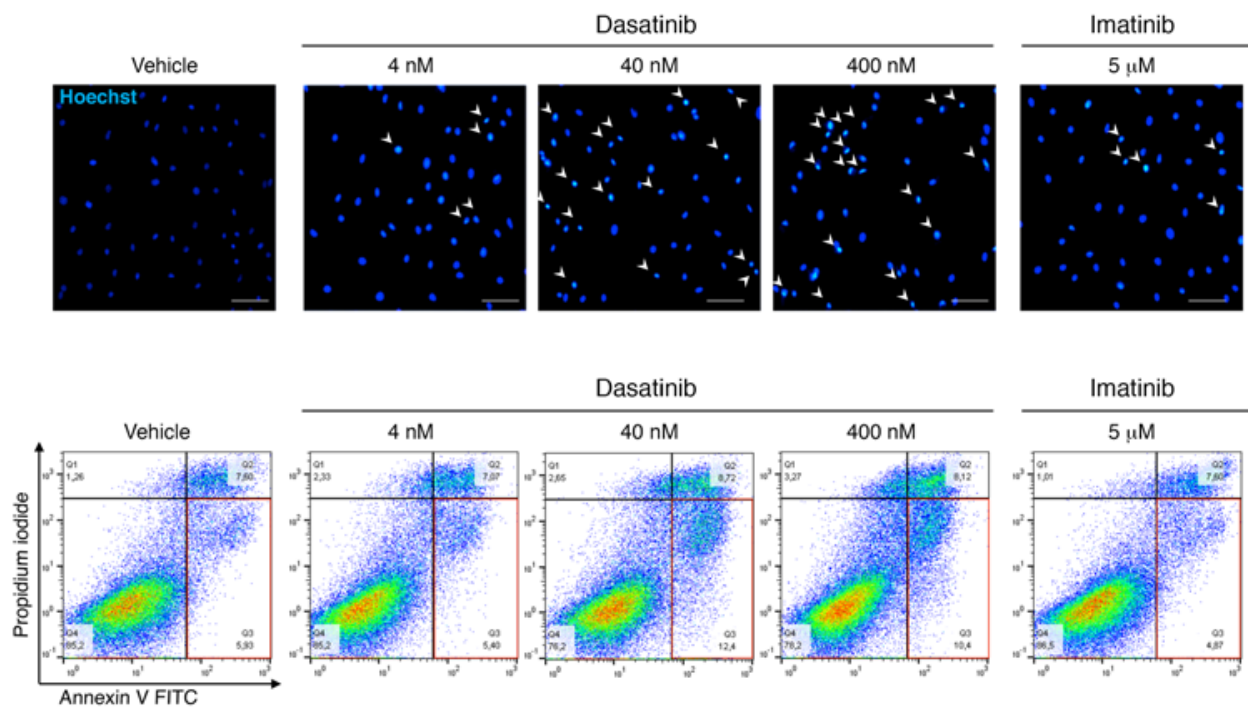

Dasatinib
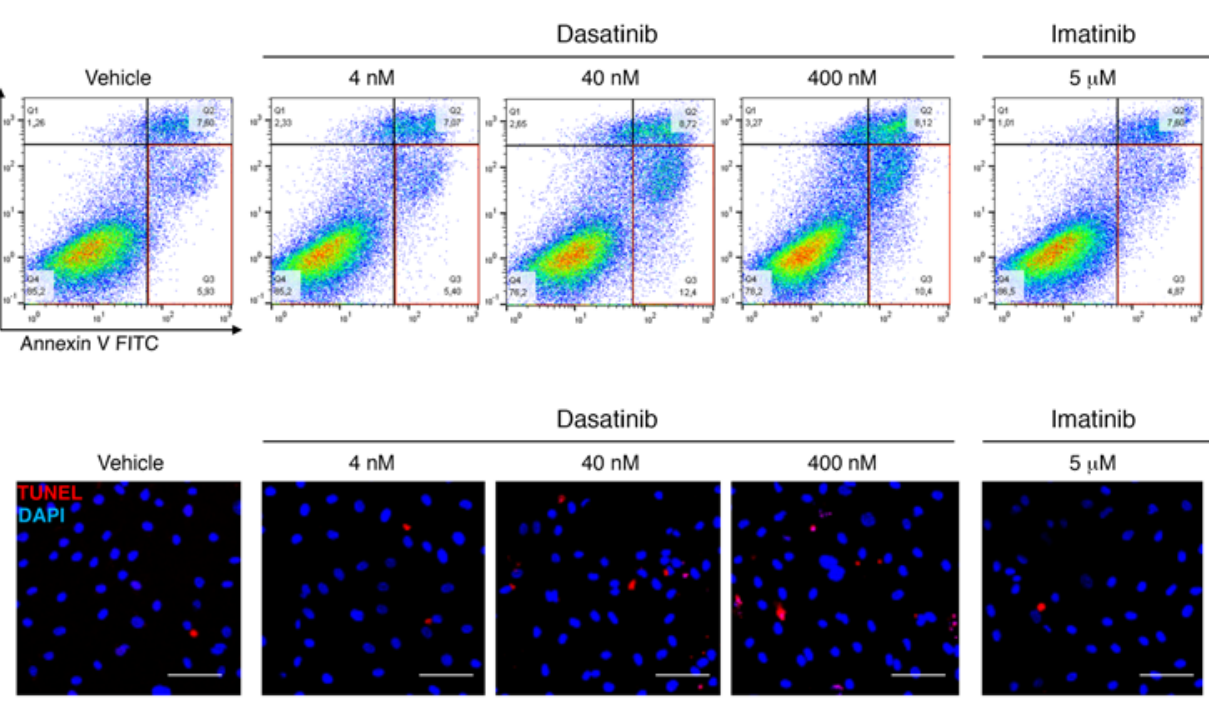

Dasatinib

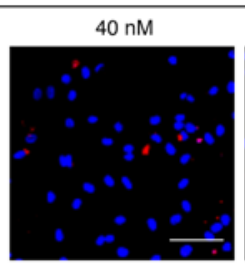

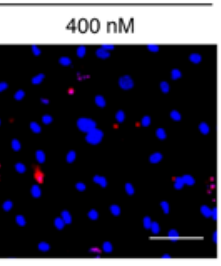

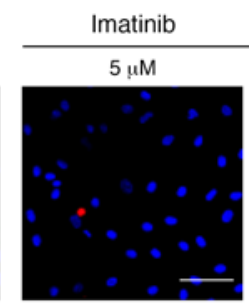

Figure 3. Dasatinib-induced apoptosis of human pulmonary ECs in vitro. (A) Representative images of human pulmonary EC monolayers (passage $<5$ ) 2 hours after treatment with vehicle, dasatinib, or imatinib. (B) Determination of caspase-3/7 activity in human pulmonary ECs treated with vehicle, dasatinib, or imatinib. (C) Quantification of the percentage of apoptotic cells using Hoechst 33342 staining and representative images. (D) Quantification of annexin $\mathrm{V}$ and propidium iodide (PI) dual labeling in human pulmonary ECs treated with vehicle, dasatinib, or imatinib and representative FACS dot plots. (E) Quantification of TUNEL-positive cells in human pulmonary ECs treated with vehicle, dasatinib, or imatinib and representative images. Horizontal lines display the mean $\pm \operatorname{SEM}(n=5)$. ${ }^{*} P<0.05,{ }^{* *} P<0.01,{ }^{* * *} P<0.0001$ vs. vehicle-treated cells; ${ }^{* \#} P<0.01$, ${ }^{* \# \#} P<0.0001$ vs. human pulmonary ECs treated with $400 \mathrm{nM}$ dasatinib. Scale bars: $20 \mu \mathrm{m}$.

Altogether, our in vivo findings demonstrate that dasatinib (1x and 10x) induces pulmonary EC dysfunction leading to a blunted HPV, to an abnormal expression of adhesion molecules, and to ER stress, a phenomenon not found with imatinib.

Dasatinib-induced apoptosis in cultured human pulmonary ECs, independent of its action on Src family kinases. We next assessed dasatinib-induced pulmonary EC dysfunction by studying its direct effect on primary cultures of human pulmonary ECs in vitro. We treated cultured pulmonary ECs, generated from human lung specimens, with either vehicle, dasatinib (doses of dasatinib were chosen based on its pharmacokinetic profile: 4,40 , and $400 \mathrm{nM}$ ), or imatinib as previously described (19-24). Interestingly, we showed that in vitro dasatinib treat- ment led to pulmonary EC apoptosis in a dose-dependent manner (Figure 3). A significant increase in apoptotic cells was found at $40 \mathrm{nM}$ and was even more pronounced at $400 \mathrm{nM}$ as assessed by caspase- $3 / 7$ activity (Figure $3 \mathrm{~B}$ ), Hoechst staining (Figure 3C), staining with annexin $\mathrm{V}$ and propidium iodide by flow cytometry (Figure 3D), and TUNEL (Figure 3E), in contrast to vehicle- and imatinib-treated cells. Consistently with these findings, we noted a substantial decrease in cell number at 16 hours in dasatinib-treated cells in contrast to vehicle- and imatinib-treated cells (Supplemental Figure 3).

We then investigated the underlying molecular mechanisms responsible for dasatinib-induced apoptosis in pulmonary ECs. Since it is known that dasatinib strongly inhibits the Src kinases, 
A

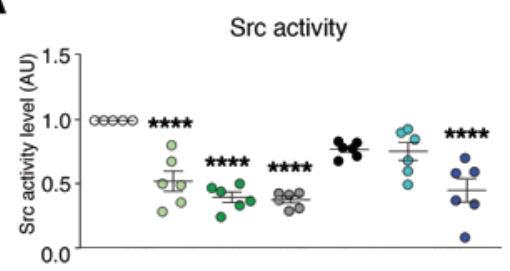

$\mathbf{B}$
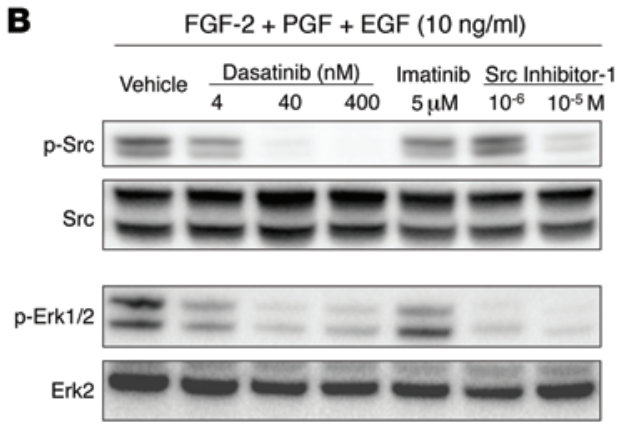

O Vehicle

O Dasatinib, $4 \mathrm{nM}$

- Dasatinib, $40 \mathrm{nM}$

O Dasatinib, $400 \mathrm{nM}$

- Imatinib, $5 \mu \mathrm{M}$

O Src Inhibitor-1, $10^{-6} \mathrm{M}$

- Src Inhibitor-1, $10^{-5} \mathrm{M}$
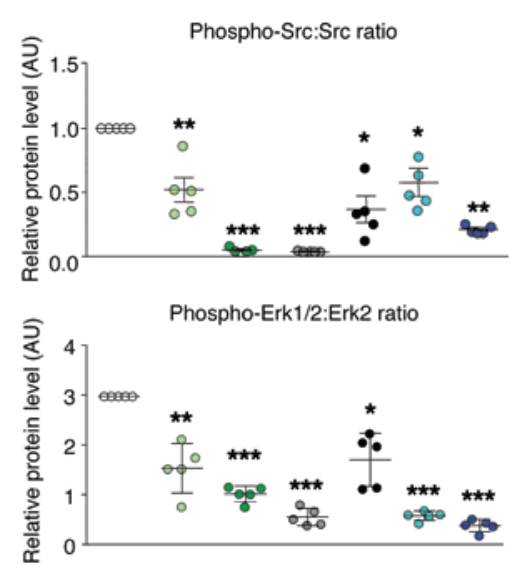

C

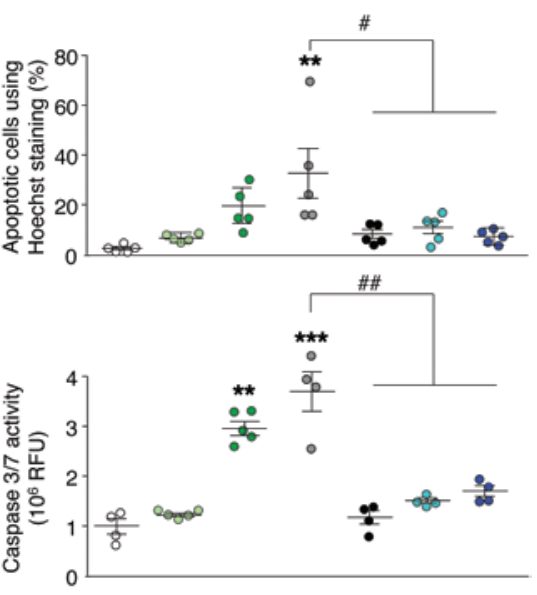

E

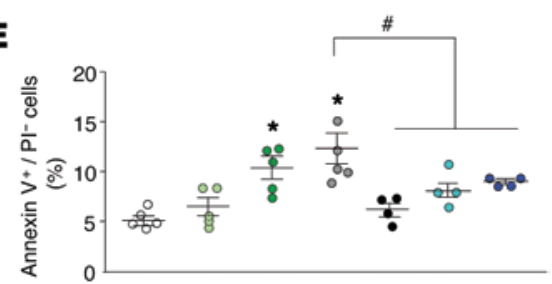

Figure 4. Dasatinib-induced apoptosis in cultured human pulmonary ECs is independent of its action on Src family kinases. (A) Quantification of the activity of Src directly in human pulmonary ECs treated with vehicle, dasatinib, imatinib, or Src inhibitor-1. (B) Representative Western blots and quantification of the phospho-Src/Src ratio and phospho-ERK1/2/ERK2 ratio in human pulmonary ECs exposed to a growth factor cocktail and treated with vehicle, dasatinib, imatinib, or Src inhibitor-1. (C-E) Quantification of pulmonary EC apoptosis using Hoechst 33342 (C), caspase-3/7 activity (D), and double staining with annexin $V$ and propidium iodide (PI) by flow cytometry $(E)$ in pulmonary ECs treated with vehicle, dasatinib, imatinib, or Src inhibitor-1. Horizontal lines display the mean \pm SEM $(n=4-6) .{ }^{*} P<0.05,{ }^{* *} P<0.01,{ }^{* *} P<0.001$, ${ }^{* * *} P<0.0001$ vs. vehicle-treated cells; ${ }^{*} P<0.05$, ${ }^{* \#} P<0.01$ vs. human pulmonary ECs treated with $400 \mathrm{nM}$ dasatinib. FGF-2, fibroblast growth factor 2 (basic); PDGF, platelet-derived growth factor; ECF, epidermal growth factor.

we first examined the role of the Src family tyrosine kinase in the dasatinib-induced pulmonary EC apoptosis. We used the selective inhibitor of Src family tyrosine kinase Src inhibitor-1, which suppresses Src kinase activity in pulmonary ECs exposed to a growth factor cocktail (Figure 4, A and B). By treating cultured ECs isolated from human control lungs with Src inhibitor-1, we did not observe any apoptotic effect as assessed by the number of apoptotic cells assessed by Hoechst staining (Figure 4C), caspase-3/7 activity (Figure 4D), and annexin V/propidium iodide coimmunostaining (Figure 4E).

Thus, these results strongly support that dasatinib-induced pulmonary EC apoptosis is independent of Src inhibition.

Dasatinib induces apoptosis in cultured human pulmonary ECs through mitochondrial ROS production. Since oxidative stress is an important determinant of endothelial dysfunction and vascular injury and intracellular ROS potently stimulate EC adhesion molecule expression $(25,26)$, we hypothesized that dasatinib may lead to increased intracellular ROS in pulmonary ECs, thereby inducing EC apoptosis. Double staining on rat lungs with the endothelial marker vWF and 8-oxo-2'-deoxyguanosine (8-oxo-dG), a marker of DNA oxidation, revealed that in vivo dasatinib treatment led to an increased expression of 8-oxo-dG in pulmonary ECs in comparison with vehicle- and imatinib-treated rats (Figure 5A). Consistently with a previous study (27), we also found significant increased levels of oxidized proteins (protein carbonyl products) and glutathione in lung homogenates from rats treated with dasatinib compared with vehicle or imatinib (Figure 5B), in a dose-dependent manner. More- over, intracellular ROS were also measured by 2 different detection methods, namely fluorogenic probes (CellRox) and dihydroethidium, in cultured human pulmonary ECs, confirming a dose-dependent dasatinib-induced increase in endothelial ROS (Figure 5C).

To test whether this increased ROS production was due to an intracellular altered balance between pro- and antioxidant enzymes, we measured the mRNA levels of NADPH oxidase 4 (NOX4), NO synthase 2 (NOS2), heme oxygenase 1 (HMOX1), GA-binding protein $\alpha$ chain (GABPA), flavin-containing monooxygenase 2 (FMO2), catalase (CAT), glutathione peroxidase 1 (GPX1), and superoxide dismutase 1 (SOD1) in rat lung homogenates. We found no difference between in vivo vehicle, dasatinib, and imatinib treatments, suggesting that the increased ROS that we observed in vivo and in vitro were due to an increased dasatinib-induced production by pulmonary ECs (Figure 5D). We then hypothesized that dasatinib induces increased mitochondrial ROS production in pulmonary ECs. To test this hypothesis, we subjected cultured pulmonary ECs to a mitochondrial ROS fluorescent probe and stained them with a fluorescent mitochondrial marker. We found that dasatinib in vitro treatment led to a dose-dependent increase in mitochondrial ROS production (Figure 5E). We next examined the functional importance of this ROS production. We found that dasatinib-induced ROS production caused pulmonary EC apoptosis, in contrast to vehicle- and imatinib-treated ECs: indeed, preincubation of pulmonary ECs for 16 hours with the antioxidative agent $N$-acetylcysteine markedly inhibited dasatinib-induced pulmonary EC apoptosis (Figure 5, F-H). 
Overall, our findings provide in vitro evidence that dasatinib stimulates mitochondrial ROS production in a dose-dependent manner, which leads to pulmonary EC apoptosis in vitro.

Patients with CML treated with dasatinib display increased serum sICAM-1, sVCAM-1, and sE-selectin concentrations. Finally, we validated our results supporting that dasatinib could have a potential role as an inducer of pulmonary endothelial toxicity in humans. We measured circulating levels of SICAM-1, sVCAM-1, and sEselectin in patients with chronic myelogenous leukemia (CML) (without PAH) at diagnosis, in CML patients treated with TKIs for less than 3 months, either with imatinib or dasatinib, and in healthy subjects. We found that CML patients treated with dasatinib displayed higher levels of sICAM-1, sVCAM-1, and sE-selectin compared with CML patients at diagnosis, imatinib-treated CML patients, or healthy subjects (Figure 6). These results, consistently with our in vivo and in vitro data, support the notion that dasatinib induces endothelial dysfunction and therefore may contribute to dasatinib-induced PAH.

\section{Discussion}

Our present report demonstrates that pretreatment of rats with dasatinib increases susceptibility to experimental $\mathrm{PH}$, which closely mimicks the findings observed in humans. We studied rats treated or not treated with dasatinib or imatinib, with or without exposure to MCT or chronic hypoxia, 2 known inducers of $\mathrm{PH}$. We performed in vitro studies on primary early-passage cultures of ECs isolated from human lung tissues. Interestingly, we found that dasatinib leads to increased levels of circulating markers of endothelial dysfunction or vascular injury in humans and rodents and induction of chronic ER stress and oxidative stress, in contrast to imatinib. We also found in vitro that dasatinib induces pulmonary EC apoptosis in a dose-dependent fashion, through production of ROS. In an effort to identify the mechanism(s) responsible for the pulmonary vascular safety issues in dasatinib-treated patients, we examined serum specimens from CML patients treated with dasatinib or imatinib. We showed significant elevations of circulating sICAM-1, sVCAM-1, and $\mathrm{sE}$-selectin in the serum of CML patients treated with dasatinib compared with CML patients treated with imatinib, further supporting the notion that dasatinib could lead to vascular injury and EC dysfunction and damage.

It is known that dasatinib is a cause of drug-induced $\mathrm{PAH}$, in addition to evidence supporting its beneficial effects in experimental models $(8,28)$ : between November 2006 and September 2010, 9 cases treated with dasatinib at the time of PAH diagnosis were identified in the French PH Registry (9). These published cases of dasatinib-associated $\mathrm{PAH}$ were characterized by rapid clinical, functional, and hemodynamic improvements within 4 months of dasatinib discontinuation in 8 patients (9). However, after a median follow-up of 9 months, most patients did not demonstrate complete recovery, and 2 patients died. Today, more than 100 cases of dasatinib-induced PAH have been submitted for European pharmaceutical vigilance, and key questions remain regarding the long-term evolution of $\mathrm{PAH}$ hemodynamics and the mechanism(s) involved. As a result, we lack effective markerbased screening strategies and mechanism-based treatment options for PAH occurring in dasatinib-treated patients.
Our results, showing a direct endothelial toxicity induced by dasatinib, strongly support the hypothesis that dasatinib may increase the risk of developing PAH in the presence of an as-yet unrecognized second "hit" (i.e., genetic and/or environmental factors) (Supplemental Figure 4). Indeed, increased pulmonary EC injury and apoptosis are known to act as triggers to initiate PAH (29-31). Consistent with this notion, we show that pretreatment of rats with dasatinib (at a dose of $1 \times$ or $10 \times$ ), but not imatinib, increases MCT- and chronic hypoxia-induced PH. In contrast, a beneficial effect of imatinib in our MCT- and chronic hypoxiainduced $\mathrm{PH}$ was noted, an observation that is consistent with findings of previous studies $(22,28,30,32)$. Overall, our work therefore supports the notion that a dose-response relationship may exist between the dose of dasatinib and the vascular safety issues observed in dasatinib-treated patients. However, further studies are clearly needed to validate this idea. In addition, our observations obtained with dasatinib pretreatments in rats also suggest that patients treated with dasatinib are likely more susceptible to develop PH in response to other environmental risk factors, such as hypoxia or certain other toxins.

Because plasma dasatinib pharmacokinetics varies over time after oral administration with a peak plasma concentration ( 90 nM) 0.5-3 hours after oral administration (33), inhibition of different receptor and/or nonreceptor tyrosine kinases can be responsible for the vascular safety issues in dasatinib-treated patients. Indeed, dasatinib is rapidly absorbed and distributed in the extravascular space and is highly protein bound ( 94\%), which results in a large volume of distribution of $2,505 \mathrm{~L}$ and a half-life of 3-5 hours (34). Moreover, dasatinib is extensively metabolized in the liver, predominantly by cytochrome $\mathrm{P} 450$ 3A4 (CYP3A4) $(34,35)$. Therefore, to unravel the mechanism(s) responsible for the vascular safety issues in dasatinib-treated patients, we have used primary early-passage $(<5)$ cultures of human pulmonary ECs exposed or not exposed to increasing doses of dasatinib. In addition, imatinib that reaches concentrations of approximately $5 \mu \mathrm{M}$ in patient plasma was used as a TKI control in our experiments $(33,36)$. Our data show that dasatinib treatment induces pulmonary EC apoptosis in a dose-dependent manner, whereas imatinib has no apparent effect. In order to identify the specific receptor and/or nonreceptor tyrosine kinases responsible for this phenomenon, we focused our attention on Src kinase. Indeed, Nagaraj et al. (37) have demonstrated that inhibition of Src with $30 \mu \mathrm{M}$ of PP2 or with $100 \mu \mathrm{M}$ of dasatinib causes a substantial increase in the PAP of isolated perfused mouse lungs via the control of members of the 2-pore domain potassium channel family in human pulmonary artery smooth muscle cells (37). However, herein, we found that HPV was significantly attenuated in rats treated with a high dose of dasatinib. Consistent with our findings, we demonstrate that dasatinib-mediated apoptosis in pulmonary ECs is not dependent on Src kinase inhibition. Two-pore domain potassium channels are known also to be potential regulators of apoptotic cell death; thus we cannot exclude that their inhibition may also partly contribute to dasatinib-mediated apoptosis in pulmonary ECs (38-42). In contrast to imatinib, we interestingly demonstrate that dasatinib treatment causes a rapid and dose-dependent increase in ROS generation that may cause the 
A

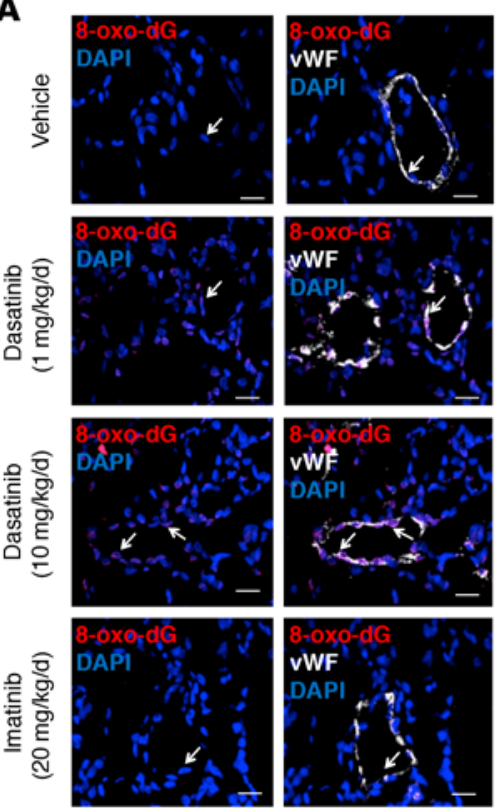

D

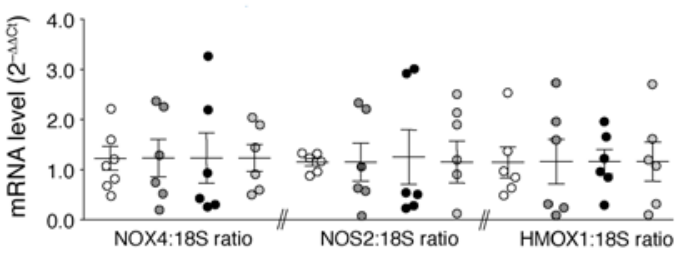

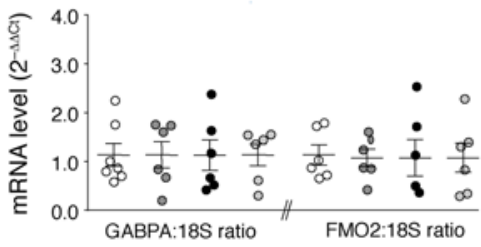

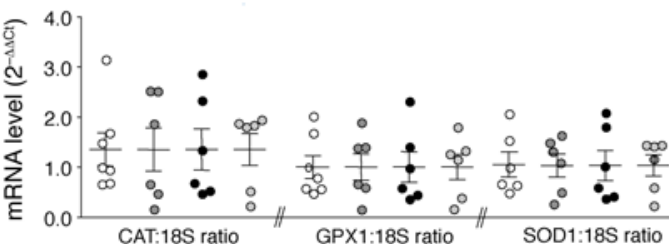

$\mathbf{F}$

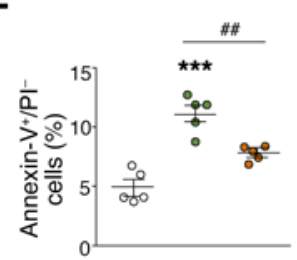

G

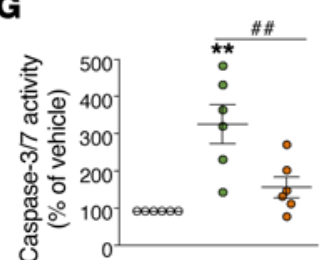

B

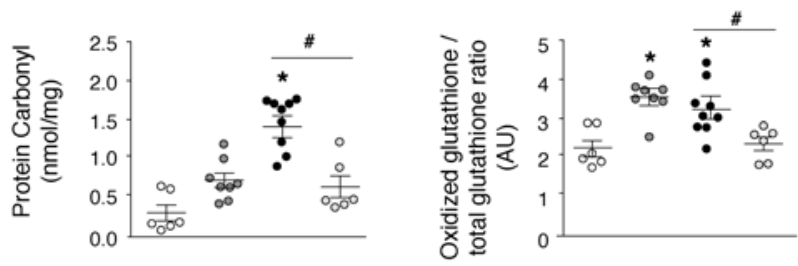

Vehicle

- Dasatinib, $1 \mathrm{mg} / \mathrm{kg} / \mathrm{d}$

- Dasatinib, $10 \mathrm{mg} / \mathrm{kg} / \mathrm{d}$

O Imatinib, $20 \mathrm{mg} / \mathrm{kg} / \mathrm{d}$
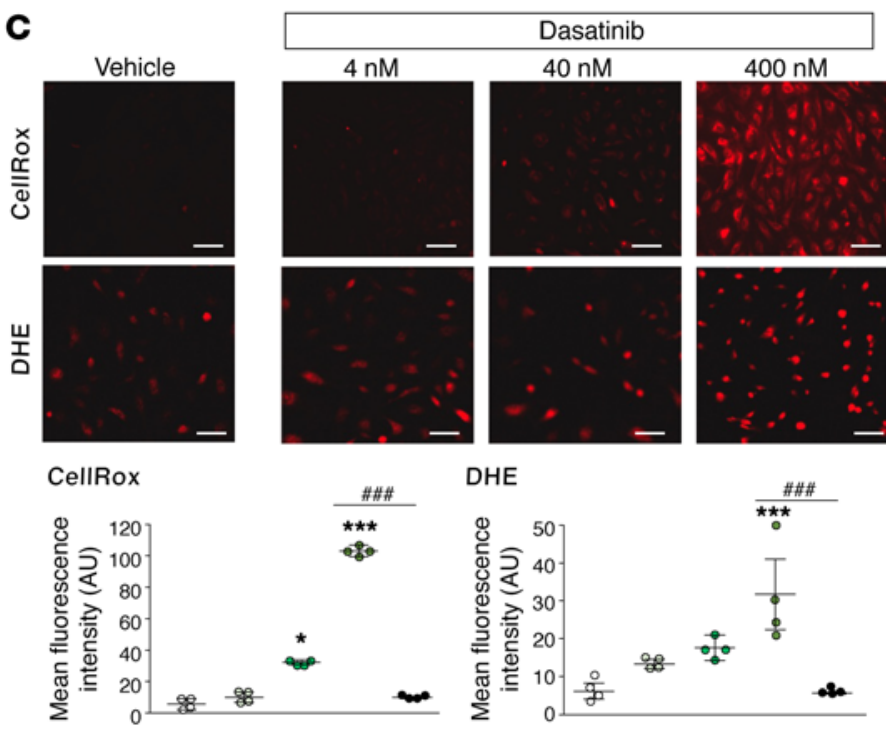

O Vehicle

O Dasatinib, $4 \mathrm{nM}$

- Dasatinib, $40 \mathrm{nM}$

- Dasatinib, $400 \mathrm{nM}$

- Imatinib, $5 \mu \mathrm{M}$

- Dasatinib, $400 \mathrm{nM}$ $+\mathrm{NAC}, 5 \mathrm{mM}$
E

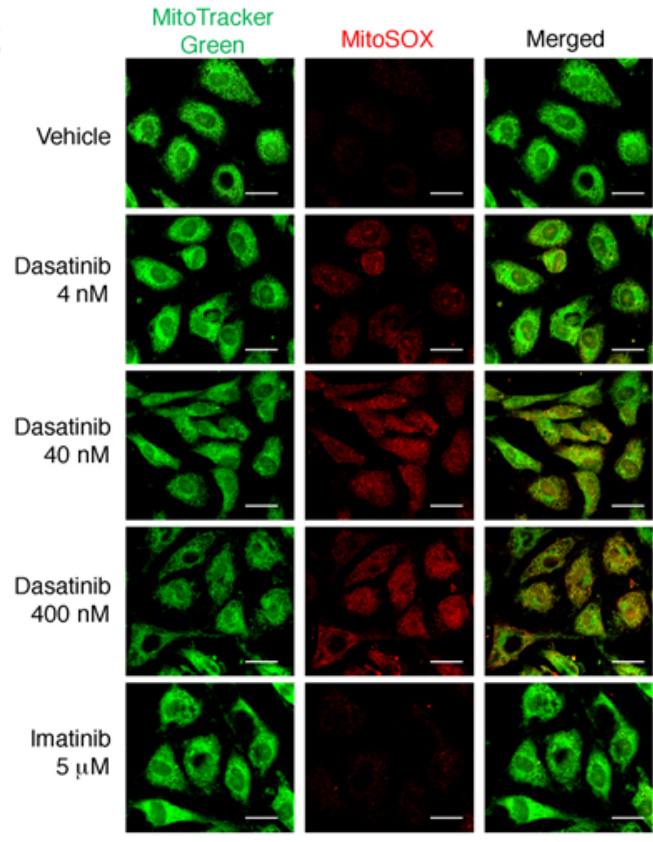

H

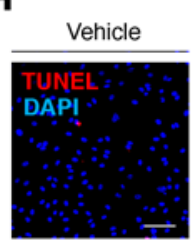

Dasatinib Dasatinib, $400 \mathrm{nM}$

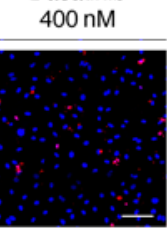

+ NAC, $5 \mathrm{mM}$

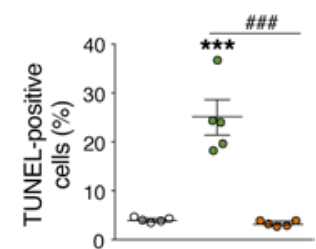


Figure 5. Dasatinib stimulates mitochondrial ROS production in vivo and in vitro. (A) Representative images obtained by confocal microscopic analyses and double labeling with 8-0xo-2'-deoxyguanosine (8-oxo-dG) and the endothelial-specific marker VWF in lungs from vehicle-, dasatinib-, and imatinib-treated rats. (B) Quantifications of the oxidized glutathione/ total glutathione ratio and of the protein carbonyl products in lungs and serum of vehicle-, dasatinib-, and imatinib-treated rats, respectively. (C) Representative images and quantification of intracellular ROS generation using fluorogenic probes (CellRox) and dihydroethidium (DHE) in pulmonary ECs treated 30 minutes with vehicle, dasatinib, or imatinib. (D) Gene expression analysis of pro-oxidative enzymes (NADPH oxidase 4 [NOX4], NO synthase 2 [NOS2], heme oxygenase 1 [HMOX1], GA-binding protein $\alpha$ chain [GABPA], flavin-containing monooxygenase 2 [FMO2]) and antioxidative enzymes (catalase [CAT], glutathione peroxidase 1 [GPX1], superoxide dismutase 1 [SOD1]) in lungs of rats treated with vehicle, dasatinib, and imatinib. (E) Mitochondria were labeled with MitoTracker Green (green) and mitochondrial ROS with MitoSOX Red (red). (F-G) Quantitative FACS analysis of the annexin $\mathrm{V}$ and propidium iodide (PI) dual labeling and quantification of caspase-3/7 activity in pulmonary ECs pretreated or not for 16 hours with $\mathrm{N}$-acetylcysteine (NAC) at $5 \mathrm{mM}$ and exposed or not to $400 \mathrm{nM}$ dasatinib. (H) Representative images and quantification of the TUNEL staining in pulmonary ECs pretreated or not for 1 hour with $\mathrm{NAC}$ at $5 \mathrm{mM}$ and exposed or not to $400 \mathrm{nM}$ dasatinib. Horizontal lines display the mean \pm SEM $(n=4-9) .{ }^{*} P<0.05,{ }^{* *} P<0.01,{ }^{* *} P<0.001$ vs.

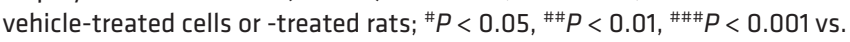
pulmonary ECs treated with $400 \mathrm{nM}$ dasatinib or rats treated with dasatinib (10x). Scale bars: $20 \mu \mathrm{m}$.

dose-dependent induction of apoptosis. The fact that there is significant interindividual variation in responses and susceptibility to oxidative stress together with the presence or absence of another environmental risk factor for PAH may partly explain the incidence of PAH occurring in patients exposed to dasatinib. In addition, individual patient variability in absorption, distribution, and metabolism as well as genetic or demographic differences between individuals could also impact on this vascular safety issue observed in dasatinib-treated patients. Substantial work remains to be done to better understand the mechanisms by which dasatinib leads to generation of mitochondrial ROS to protect the pulmonary endothelium.

Together, our work underscores that dasatinib, in contrast to imatinib, causes pulmonary vascular damage and aggravates experimental $\mathrm{PH}$ through production of mitochondrial ROS, providing promising perspectives for a better understanding and management of patients treated with dasatinib.

\section{Methods}

Study population. Blood samples were taken from CML patients at diagnosis (SPIRIT, ClinicalTrials.gov NCT00219739) or CML patients treated with a standard dose of dasatinib or imatinib for less than 3 months (OPTIM Dasatinib, EudraCT 2008-006854-17, and OPTIM Imatinib, EudraCT 2010-019568-35) (Supplemental Table 1). All patients gave informed consent before the study.

Animals and in vivo treatment. Young male Wistar rats (100 g; Janvier Labs) were treated daily with a single i.p. injection of dasatinib, imatinib, or vehicle (DMSO) for 4 weeks (group A). An additional group of rats were pretreated 1 week with dasatinib $(1 \mathrm{or} 10 \mathrm{mg} / \mathrm{kg} / \mathrm{d}$ ), imatinib (20 mg/kg/d), or vehicle (DMSO) and studied either 3 weeks after a single s.c. injection of MCT $(40 \mathrm{mg} / \mathrm{kg}$; Sigma-Aldrich) or vehicle, or 3 weeks after chronic exposure to hypoxia (10\% oxygen) or in room air (group B). A sensible ultra-performance liquid chromatography-tandem mass spectrometry method was used for the quantification of dasatinib active metabolite in rat plasma (43). Animals were anesthetized with isoflurane. A polyvinyl catheter was introduced into the right jugular vein and pushed through the right ventricle into the pulmonary artery. Cardiac output in rats was measured using the thermodilution method. After measurement of hemodynamic parameters, the thorax was opened and the left lung immediately removed and frozen. The right lung was fixed in the distended state with formalin buffer. The right ventricular hypertrophy was assessed by the Fulton index (weight ratio of right ventricle and [left ventricle + septum]), and the percentage of wall thickness ([2 $\times$ medial wall thickness/external diameter] $\times 100$ ) and of muscularized vessels was calculated as previously described $(19,20,22,23)$.

Assessment of HPV. Pulmonary vascular reactivity was assessed in anesthetized rats that were first ventilated with room air (baseline) and then exposed to 10 minutes of ventilation with hypoxic gas mixture (10\% oxygen, $90 \%$ nitrogen). Three similar successive hypoxic challenges were repeated in each rat.

Isolation and culture of human pulmonary ECs. Human pulmonary ECs were isolated and cultured as previously described (19-24). For the in vitro studies, we used lung specimens obtained during lobectomy or pneumonectomy for localized lung cancer in control subjects. Preoperative echocardiography was performed in these control patients to rule out $\mathrm{PH}$. The lung specimens from the controls were collected at a distance from the tumor foci. The absence of tumoral infiltration was retrospectively established in all tissue sections by the histopathological analysis.
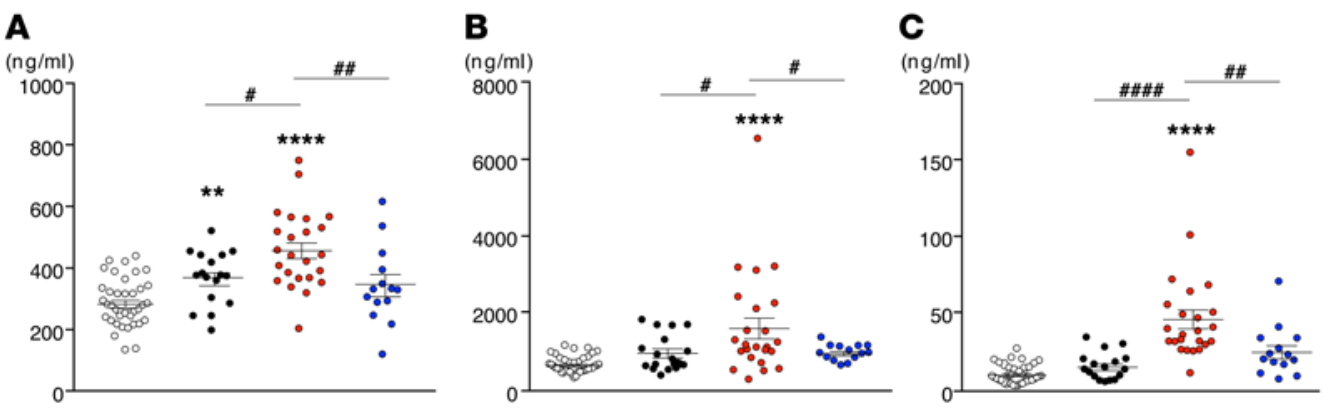

Figure 6. Patients with CML treated with dasatinib display increased serum concentrations of sICAM-1, sVCAM-1, and sE-selectin. Quantifications of sICAM-1 (A), sVCAM-1 (B), and sE-selectin (C) in CML patients at diagnosis $(n=17)$, in CML patients treated with dasatinib or imatinib after less than 3 months of therapy ( $n=24$ and 14 , respectively), and in healthy subjects $(n=39)$. Horizontal lines display the mean $\pm \operatorname{SEM} .{ }^{* *} P<0.01,{ }^{* * * *} P<0.0001$ vs.

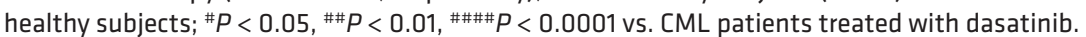


The isolated pulmonary ECs were strongly positive for acetylated LDL coupled to Alexa 488, vWF, and CD31 and for Ulex europaeus agglutinin- 1 and negative for $\alpha$-smooth muscle actin. The cells were used at a passage less than 5 .

Real-time quantitative PCR. Levels of lung mRNA encoding NOX4, NOS2, HMOX1, GABPA, FMO2, CAT, GPX1, and SOD1 were measured by real-time quantitative PCR according to the method previously described $(23,44,45)$.

Western blot, ELISA, and immunostaining. The primary antibodies used in this study are listed in Supplemental Table 2. Cells and tissues were homogenized and sonicated in RIPA buffer containing protease and phosphatase inhibitors, and $30 \mu \mathrm{g}$ of protein was used to detect phospho-ERK1/2, ERK2, XBP1, GRP78, ATF6, eIF2 $\alpha$ (Santa Cruz Biotechnology), phospho-Src, Src, phospho-eIF2 $\alpha$ (Cell Signaling Technology), and $\beta$-actin (Sigma-Aldrich) as previously described (19-24). The activity of Src in $30 \mu \mathrm{g}$ of cell lysates was determined using the Src kinase assay kit (CycLex Co.). Concentrations of sICAM-1, sVCAM-1, and sE-selectin in human serum were evaluated using a specific ELISA kit (R\&D Systems) according to the manufacturer's instructions. Concentrations of sICAM-1, sVCAM-1, sE-selectin, and protein carbonyl in rat serum and glutathione in lung homogenates were evaluated using ELISA kits for rat sICAM-1 (BosterBio), sVCAM-1, and sE-selectin (Cusabio), for protein carbonyl (Cell Biolabs), and for glutathione (Enzo Life Sciences) according to the manufacturers' instructions. IHC and immunocytofluorescent staining for ICAM-1, E-selectin, $\alpha$-smooth muscle actin, CD68, and 8-oxo-dG (Santa Cruz Biotechnology), VCAM-1 (Abcam), and CD3 and CD45 (BD Pharmingen) were performed as previously described $(19,20,22,23)$.

Apoptosis detection. Cells were counted using pictures taken with a microscope. The cells were counted per field. Hoechst 33342 (Thermo Fisher Scientific) was used to detect apoptosis. Cells (10,000 cells/ $\mathrm{ml}$ ) were seeded in a 4 -well Lab-Tek Chamber Slide and treated with dasatinib $(4,40,400 \mathrm{nM}$ in media with $1 \%$ serum $)$ and imatinib $(5 \mu \mathrm{M}$ in media containing $1 \%$ serum) for 2 hours at $37^{\circ} \mathrm{C}$. Cells were fixed with ice-cold methanol for 10 minutes. The pictures were taken with a Nikon Eclipse 80i microscope (Nikon Instruments Europe BV). Nuclei were scored as either normal (uncondensed chromatin with bright blue fluorescence) or apoptotic (highly condensed chromatin with bright blue fluorescence).

To measure activity of caspase-3/7 using an Apo-ONE kit (Promega), cells were seeded in a 96-well plate. After treatment with dasatinib and imatinib for 2 hours in media containing 1\% serum, the specific reagents were added in every well as recommended by the manufacturer. For $\mathrm{N}$-acetylcysteine treatment, cells were pretreated before being exposed to $400 \mathrm{nM}$ dasatinib. The fluorescence was monitored by EnVision (PerkinElmer). The mean of fluorescence is proportional to the apoptosis.

The annexin V-FITC assay was used to validate the presence of apoptosis. Floating cells were collected with adherent cells harvested by trypsin/EDTA treatment and stained with annexin V-FITC (BD Biosciences), then analyzed by flow cytometry MACSQuant (Miltenyi Biotec). In each sample, at least 10,000 cells were counted by FACS analysis.

In addition, we conducted TUNEL staining using the ApopTag red in situ apoptosis detection kit (Chemicon) according to the manufacturer's instructions. The number of TUNEL-positive nuclei per total number of nuclei was counted.

Fluorescent detection of ROS. The superoxide indicator dihydroethidium (DHE; Thermo Fisher Scientific) was used to evaluate intra- cellular ROS in cultured human pulmonary ECs. Cells (10,000 cells/ $\mathrm{ml}$ ) were seeded in a Lab-Tek Chamber Slide and treated with dasati$\mathrm{nib}(4,40,400 \mathrm{nM}$ in media containing $1 \%$ serum $)$ and imatinib $(5 \mu \mathrm{M}$ in media containing $1 \%$ serum) for 30 minutes at $37^{\circ} \mathrm{C}$. DHE (10 $\left.\mu \mathrm{M}\right)$ was added and incubated for 20 minutes at $37^{\circ} \mathrm{C}$. After incubation, the cells were washed twice with PBS, and the Lab-Tek Chamber Slide was mounted with ProlonGold antifade reageant (Thermo Fisher Scientific). The mean of fluorescence is proportional to the intracellular superoxide production.

CellRox (Thermo Fisher Scientific) was used to estimate a cytoplasmic superoxide production in cultured pulmonary ECs. Cells (10,000 cells $/ \mathrm{ml}$ ) were seeded in a Lab-Tek Chamber Slide and treated with dasatinib (4, 40, $400 \mathrm{nM}$ in media containing 1\% serum) and imatinib (5 $\mu \mathrm{M}$ in media with $1 \%$ serum) for 30 minutes at $37^{\circ} \mathrm{C}$. CellRox $(10$ $\mu \mathrm{M})$ was added and incubated for 30 minutes at $37^{\circ} \mathrm{C}$. After incubation, the cells were washed with PBS, and the Lab-Tek Chamber Slide was mounted with ProlonGold. The mean of fluorescence is proportional to the cytoplasmic superoxide production. Fluorescence was monitored with a Nikon Eclipse $80 \mathrm{i}$ microscope.

MitoSOX Red (Thermo Fisher Scientific) was used to detect mitochondrial superoxide production. Briefly, 5.0 $\mu \mathrm{M}$ MitoSOX was used in the presence of a mitochondrial-selective fluorescent label MitoTracker Green (200 nM; Thermo Fisher Scientific) for 25 minutes. Subsequently, cells were washed in HBSS and mounted with ProlonGold. Images were taken using an LSM700 confocal microscope (Zeiss).

Statistics. Statistical significance was tested using the nonparametric Mann-Whitney $U$ test or 2-way ANOVA with Bonferroni post hoc tests. Significant difference was assumed at a $P$ value less than 0.05 . Continuous data are expressed as mean \pm SEM of 3 independent experiments, each performed in triplicate or quintuplicate. A $P$ value less than 0.05 was considered statistically significant. Analyses were performed using GraphPad Prism 5.0.

Study approval. All animals were treated in accordance with the Guide for the Care and Use of Laboratory Animals as adopted by INSERM, and approval was granted by the Ethics Committee of Université Paris-Sud, Le Plessis-Robinson, France. In addition, all the experiments with human specimens were approved by the local ethics committee (Comité de Protection des Personnes [CPP] Ilede-France VII, Le Kremlin-Bicêtre, France; and CPPs from Lille, Poitiers, and Bordeaux, France). All patients gave informed consent before the study.

\section{Author contributions}

CG, AH, CP, AS, LT, RT, CS, MLH, and EMJ performed experiments and analyzed data. CG, LT, and CP designed and performed experiments and analyzed data. DM, AH, OS, GS, and $\mathrm{MH}$ analyzed the cases of dasatinib-induced $\mathrm{PAH}$ from the French Registry. SB, BM, MM, and PR participated in the sample collection from CML patients treated with imatinib or dasatinib and in pharmacokinetic measurement. CG, AH, DM, and $\mathrm{MH}$ wrote the manuscript. AS, CP, LT, MLH, YT, MCC, SB, BM, MM, and $\mathrm{PR}$ read and corrected the manuscript. All authors approved the final manuscript.

\section{Acknowledgments}

The authors thank Claude Preudhomme (Centre Hospitalier Régional Universitaire de Lille, France) and François Guilhot 
(INSERM CIC 1402 Oncologie Hématologique et Thérapie Cellulaire, Centre Hospitalier Universitaire de Poitiers, France) for their help in the collection and processing of CML samples patients at diagnosis (STI571 Prospective Randomized Trial (SPIRIT), NCT00219739 [Programme Hospitalier de recherche Clinique 2002, 2006, and EudraCT 030482]). This research was supported by grants from the French National Institute for Health and Medical Research (INSERM), Université Paris-Sud, the Legs Poix (Chancellerie des Universités de Paris); the French National Agency for Research (grant ANR_12_JSV1_0004_01); the French Agence Nationale de Sécurité du Médicament (ANSM grant VIGIAPATH); and in part by the Département HospitaloUniversitaire Thorax Innovation and the LabEx LERMIT (grant
ANR-10-LABX-0033-LERMIT). YT (RESPIRE2-2013-4919) is the recipient of a European Respiratory Society/European Union RESPIRE2 Marie Curie research fellowship. AS is supported by the French PAH patient association, hypertension artérielle pulmonaire (HTAP) France. EMJ is the recipient of an Année-Recherche fellowship from Assistance Publique-Hôpitaux de Paris. CS and $\mathrm{CP}$ are supported by the French Fonds de Dotation Recherche en Santé Respiratoire-Fondation du Souffle.

Address correspondence to: Christophe Guignabert, INSERM UMR_S 999/Université Paris-Sud, Hôpital Marie Lannelongue, 133 Avenue de la Résistance, 92350 Le Plessis-Robinson, France. Phone: 33.1.40948833; E-mail: christophe.guignabert@inserm.fr.
1. Galiè N, et al. 2015 ESC/ERS Guidelines for the diagnosis and treatment of pulmonary hypertension: The Joint Task Force for the Diagnosis and Treatment of Pulmonary Hypertension of the European Society of Cardiology (ESC) and the European Respiratory Society (ERS). Endorsed by: Association for European Paediatric and Congenital Cardiology (AEPC), International Society for Heart and Lung Transplantation (ISHLT). Eur Respir J. 2015;46(4):903-975.

2. Guignabert C, Dorfmuller P. Pathology and pathobiology of pulmonary hypertension. Semin Respir Crit Care Med. 2013;34(5):551-559.

3. Guignabert C, et al. New molecular targets of pulmonary vascular remodeling in pulmonary arterial hypertension: importance of endothelial communication. Chest. 2015;147(2):529-537.

4. Guignabert C, et al. Pathogenesis of pulmonary arterial hypertension: lessons from cancer. Eur Respir Rev. 2013;22(130):543-551.

5. Huertas A, et al. Immune dysregulation and endothelial dysfunction in pulmonary arterial hypertension: a complex interplay. Circulation. 2014;129(12):1332-1340.

6. Rabinovitch M, Guignabert C, Humbert M, Nicolls MR. Inflammation and immunity in the pathogenesis of pulmonary arterial hypertension. Circ Res. 2014;115(1):165-175.

7. Tuder RM, et al. Relevant issues in the pathology and pathobiology of pulmonary hypertension. JAm Coll Cardiol. 2013;62(25 suppl):D4-D12.

8. Simonneau $\mathrm{G}$, et al. Updated clinical classification of pulmonary hypertension. J Am Coll Cardiol. 2013;62(25 suppl):D34-D41.

9. Montani D, et al. Pulmonary arterial hypertension in patients treated by dasatinib. Circulation. 2012;125(17):2128-2137.

10. Bantscheff $M$, et al. Quantitative chemical proteomics reveals mechanisms of action of clinical ABL kinase inhibitors. Nat Biotechnol. 2007;25(9):1035-1044.

11. Shaker ME, Zalata KR, Mehal WZ, Shiha GE, Ibrahim TM. Comparison of imatinib, nilotinib and silymarin in the treatment of carbon tetrachloride-induced hepatic oxidative stress, injury and fibrosis. Toxicol Appl Pharmacol. 2011;252(2):165-175.

12. Kim Y, et al. Anti-fibrotic activity and enhanced interleukin- 6 production by hepatic stellate cells in response to imatinib mesylate. Liver Int.
2012;32(6):1008-1017.

13. Sommer N, Strielkov I, Pak O, Weissmann N. Oxygen sensing and signal transduction in hypoxic pulmonary vasoconstriction. Eur Respir J. 2016;47(1):288-303.

14. Witte I, Horke S. Assessment of endoplasmic reticulum stress and the unfolded protein response in endothelial cells. Meth Enzymol. 2011;489:127-146.

15. McAlpine CS, Werstuck GH. The development and progression of atherosclerosis: evidence supporting a role for endoplasmic reticulum (ER) stress signaling. Cardiovasc Hematol Disord Drug Targets. 2013;13(2):158-164.

16. Lenna S, Han R, Trojanowska M. Endoplasmic reticulum stress and endothelial dysfunction. IUBMB Life. 2014;66(8):530-537.

17. Dromparis P, Paulin R, Sutendra G, Qi AC, Bonnet S, Michelakis ED. Uncoupling protein 2 deficiency mimics the effects of hypoxia and endoplasmic reticulum stress on mitochondria and triggers pseudohypoxic pulmonary vascular remodeling and pulmonary hypertension. Circ Res. 2013;113(2):126-136.

18. Dromparis P, Paulin R, Stenson TH, Haromy A, Sutendra G, Michelakis ED. Attenuating endoplasmic reticulum stress as a novel therapeutic strategy in pulmonary hypertension. Circulation. 2013;127(1):115-125.

19. Huertas A, et al. Leptin signalling system as a target for pulmonary arterial hypertension therapy. Eur Respir J. 2015;45(4):1066-1080.

20. Ricard N, et al. Increased pericyte coverage mediated by endothelial-derived fibroblast growth factor- 2 and interleukin- 6 is a source of smooth muscle-like cells in pulmonary hypertension. Circulation. 2014;129(15):1586-1597.

21. de Man FS, et al. Dysregulated renin-angiotensin-aldosterone system contributes to pulmonary arterial hypertension. Am J Respir Crit Care Med. 2012;186(8):780-789.

22. $\mathrm{Tu} \mathrm{L}$, et al. A critical role for $\mathrm{p} 130 \mathrm{Cas}$ in the progression of pulmonary hypertension in humans and rodents. Am JRespir Crit Care Med. 2012;186(7):666-676.

23. $\mathrm{Tu} \mathrm{L}$, et al. Autocrine fibroblast growth factor- 2 signaling contributes to altered endothelial phenotype in pulmonary hypertension. Am J Respir Cell Mol Biol. 2011;45(2):311-322.

24. Eddahibi S, et al. Cross talk between endothelial and smooth muscle cells in pulmonary hyper- tension: critical role for serotonin-induced smooth muscle hyperplasia. Circulation. 2006;113(15):1857-1864.

25. Wang GF, et al. Geniposide inhibits high glucose-induced cell adhesion through the NF-kappaB signaling pathway in human umbilical vein endothelial cells. Acta Pharmacol Sin. 2010;31(8):953-962.

26. Lutters BC, Leeuwenburgh MA, Appeldoorn CC, Molenaar TJ, Van Berkel TJ, Biessen EA. Blocking endothelial adhesion molecules: a potential therapeutic strategy to combat atherogenesis. Curr Opin Lipidol. 2004;15(5):545-552.

27. Xue T, et al. Oxidative stress is involved in Dasatinib-induced apoptosis in rat primary hepatocytes. Toxicol Appl Pharmacol. 2012;261(3):280-291.

28. Pullamsetti SS, et al. Role of Src tyrosine kinases in experimental pulmonary hypertension. Arterioscler Thromb Vasc Biol. 2012;32(6):1354-1365.

29. Goldthorpe $\mathrm{H}$, et al. Occlusive lung arterial lesions in endothelial-targeted, fas-induced apoptosis transgenic mice. Am J Respir Cell Mol Biol. 2015;53(5):712-718.

30. Guignabert C, et al. Tie2-mediated loss of peroxisome proliferator-activated receptor-gamma in mice causes PDGF receptor-beta-dependent pulmonary arterial muscularization. Am J Physiol Lung Cell Mol Physiol. 2009;297(6):L1082-L1090.

31. Long L, et al. Selective enhancement of endothelial BMPR-II with BMP9 reverses pulmonary arterial hypertension. Nat Med. 2015;21(7):777-785.

32. Schermuly RT, et al. Reversal of experimental pulmonary hypertension by PDGF inhibition. J Clin Invest. 2005;115(10):2811-2821.

33. Weisberg E, Manley PW, Cowan-Jacob SW, Hochhaus A, Griffin JD. Second generation inhibitors of BCR-ABL for the treatment of imatinib-resistant chronic myeloid leukaemia. Nat Rev Cancer. 2007;7(5):345-356.

34. Kamath AV, Wang J, Lee FY, Marathe PH. Preclinical pharmacokinetics and in vitro metabolism of dasatinib (BMS-354825): a potent oral multi-targeted kinase inhibitor against SRC and BCR-ABL. Cancer Chemother Pharmacol. 2008;61(3):365-376.

35. Korashy HM, Rahman AF, Kassem MG. Dasatinib. Profiles Drug Subst Excip Relat Methodol. 2014;39:205-237.

36. Druker BJ, et al. Efficacy and safety of a specific inhibitor of the BCR-ABL tyrosine kinase in chronic myeloid leukemia. N Engl J Med. 
2001;344(14):1031-1037.

37. Nagaraj C, et al. Src tyrosine kinase is crucial for potassium channel function in human pulmonary arteries. Eur Respir J. 2013;41(1):85-95.

38. El Hachmane MF, Rees KA, Veale EL, Sumbayev VV, Mathie A. Enhancement of TWIK-related acid-sensitive potassium channel 3 (TASK3) two-pore domain potassium channel activity by tumor necrosis factor $\alpha$. J Biol Chem. 2014;289(3):1388-1401.

39. Lauritzen I, et al. $\mathrm{K}^{+}$-dependent cerebellar granule neuron apoptosis. Role of task leak $\mathrm{K}^{+}$channels. J Biol Chem. 2003;278(34):32068-32076.
40. Trimarchi JR, Liu L, Smith PJ, Keefe DL. Apoptosis recruits two-pore domain potassium channels used for homeostatic volume regulation. Am J Physiol Cell Physiol. 2002;282(3):C588-C594.

41. Cain K, Langlais C, Sun XM, Brown DG, Cohen GM. Physiological concentrations of $\mathrm{K}^{+}$inhibit cytochrome c-dependent formation of the apoptosome. J Biol Chem. 2001;276(45):41985-41990.

42. Hughes FM, Bortner CD, Purdy GD, Cidlowski JA. Intracellular $\mathrm{K}^{+}$suppresses the activation of apoptosis in lymphocytes. J Biol Chem. 1997;272(48):30567-30576.

43. Bouchet S, et al. Simultaneous determination of nine tyrosine kinase inhibitors by $96-$ well solid-phase extraction and ultra performance LC/MS-MS. Clin Chim Acta. 2011;412(11-12):1060-1067.

44. Guignabert C, et al. Dichloroacetate treatment partially regresses established pulmonary hypertension in mice with SM22 $\alpha$-targeted overexpression of the serotonin transporter. FASEB $J$. 2009;23(12):4135-4147.

45. Le Hiress M, et al. Proinflammatory signature of the dysfunctional endothelium in pulmonary hypertension. Role of the macrophage migration inhibitory factor/CD74 complex. Am J Respir Crit Care Med. 2015;192(8):983-997. 